\title{
Discriminant Knowledge Extraction from Electrocardiograms for Automated Diagnosis of Myocardial Infarction
}

\author{
Girmaw Abebe Tadesse ${ }^{1,2, *}$, Komminist Weldemariam ${ }^{1}$, Hamza Javed ${ }^{2}$, \\ Yong $\mathrm{Liu}^{3}$, Jin $\mathrm{Liu}^{3}$, Jiyan $\mathrm{Chen}^{3}$, and Tingting $\mathrm{Zhu}^{2}$ \\ 1 IBM Research - Africa, Nairobi, Kenya \\ ${ }^{2}$ University of Oxford, Oxford, UK \\ 3 Department of Cardiology, Provincial Key Laboratory of Coronary Disease, \\ Guangdong Cardiovascular Institute, Guangzhou 510100, China \\ * Corresponding Author: girmaw.abebe.tadesse@ibm.com
}

\begin{abstract}
Visual inspection of electrocardiograms (ECGs) is a common clinical practice to diagnose heart diseases (HDs), which are still responsible for millions of deaths globally every year. In particular, myocardial infarction (MI) is the leading cause of mortality among HDs. ECGs reflect the electrical activity of the heart and provide a quicker process of diagnosis compared to laboratory blood tests. However, still it requires trained clinicians to interpret ECG waveforms, which poses a challenge in low-resourced healthcare systems, such as poor doctorto-patient ratios. Previous works in this space have shown the use of data-driven approaches to predict HDs from ECG signals but focused on domain-specific features that are less generalizable across patient and device variations. Moreover, limited work has been conducted on the use of longitudinal information and fusion of multiple ECG leads. In contrast, we propose an end-to-end trainable solution for MI diagnosis, which (1) uses 12 ECG leads; (2) fuses the leads at data-level by stacking their spectrograms; (3) employs transfer learning to encode features rather than learning representations from scratch; and (4) uses a recurrent neural network to encode temporal dependency in long duration ECGs. Our approach is validated using multiple datasets, including tens of thousands of subjects, and encouraging performance is achieved.
\end{abstract}

Keywords: Myocardial infarction · Electrocardiograms · Deep learning - Fusion

\section{Introduction}

Myocardial infarction (MI), commonly known as a heart attack, is the leading cause of cardiovascular deaths worldwide [24]. Severity of MI relates to its damaging perfusion impact resulting from blocks in coronary arteries, which interrupts the supply of oxygen and nutrients to the body [12]. It often takes hours to observe the impact of MI in blood samples as the cardiac enzymes are 


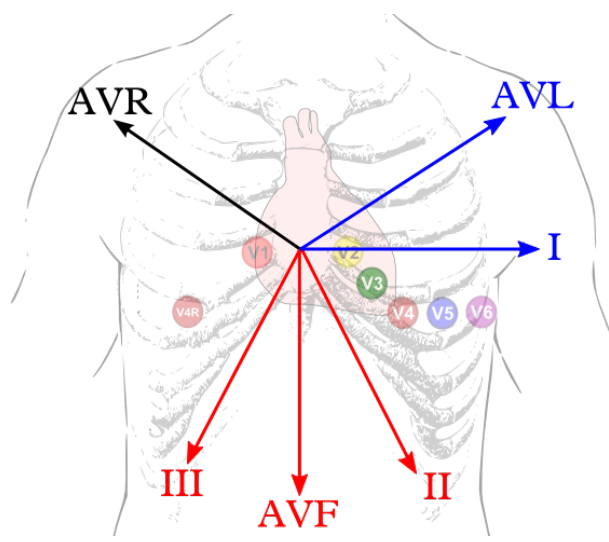

(a)

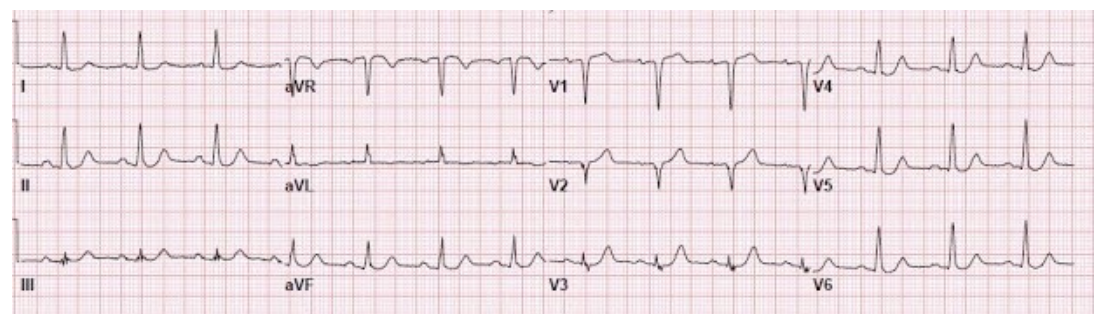

(b)

Fig. 1: (a) Conventional mounts for the 12 ECG leads, (b) Clinical visualisation of 12-lead ECG by radiologists (C)ecgpedia.org.

elevated [6]. As a result, electrocardiogram (ECG) waveform inspection (by cardiologists) has become a common clinical practice to screen MI, which partly benefits from quicker and non-invasive acquisition of these waveforms, especially in settings with less equipped laboratories to analyse blood samples. ECG waveforms represent the electrical activity of the heart from continuous polarisation and depolarisation of the atria and ventricle over time. The conventional 12-lead ECG waveforms contain the electrical signals acquired from different perspectives (see Fig. 1 (a)). An example of visualisation of these ECG signals as seen by a cardiologist is shown in Fig. 1 (b).

Inspection of ECGs provides a faster diagnosis process compared to blood sample analysis. However, the manual inspection of ECG signals is highly subjective depending on the expertise of the clinicians. This problem becomes severe in healthcare systems associated with inadequate medical experts, particularly in developing countries. Thus, the development of an intelligent diagnosis support system that can analyse ECG waveforms automatically is a practically well motivated task in healthcare $[10,20,23]$. 
The majority of existing methods for automated detection of MI from ECG traces, have focused on analysing morphological changes of the signal, such as QRS complex and P- and T-waves [16,9]. In these methods, handcrafted features are extracted from the ECG waveforms in order to make the classification using domain-expert rule-based thresholding [1], or by using data-driven machine learning (ML) models [14]. These manual feature encoding techniques necessitate extensive pre-processing steps aimed at filtering artefacts from the waveforms. Moreover, manual design (handcrafting) of features requires domain-specific expertise, resulting in a time-consuming and tedious model development process. The handcrafted features proposed to classify HDs often vary across existing works in the literature, which suggests the lack of common and generalisable features to detect HDs across variations in patient and device characteristics.

On the other hand, deep learning methods can avoid the manual feature engineering step as they can automatically learn discriminative features for a specific task. Deep learning methods have achieved state-of-the-art performance in a wide range of application domains such as natural language processing and computer vision. Clinical tasks that involve medical image analysis, have been reported to benefit from automated decision support systems that employ deep learning [15]. Existing works on HD detection from ECG readings have also reported high accuracies whilst at the same time reducing the level of expert input required [3], [8], [5], [13], [22]. Different architectures of deep learning could be considered for HD detection, but convolutional neural networks (CNNs) have been the most frequently employed [18], [5], [13]. Generally, existing deep learning methods for HD detection are limited to using only a single or a few leads of ECG waveforms, despite the fact that multiple ECG leads are understood to contain more heart-related information that can be used for improved diagnoses [18]. Moreover, effectively combining the distinctive characteristics offered by each ECG lead, e.g., using fusion techniques, is not well studied in the literature [4]. Though ECG waveforms involve periodic patterns over time, longer ECG duration might help to understand the temporal dynamics of the waveforms, e.g., through using recurrent neural networks (RNNs). In addition, existing methods are mostly validated using a small cohort of patients, which is often a bottleneck in proving the generalisability of existing methods [2], [21]. Automatic learning of features from raw ECG waveforms could also necessitate a need for larger training datasets and computational overhead.

In this paper, we propose an end-to-end trainable MI diagnosis system that employs a spectral-longitudinal model from ECG waveforms. The transfer learning approach, applied through the use of existing computer vision networks to encode discriminative features to detect MI patients, circumventing the need to manually craft features. In order to utilise the multi-perspective information available in conventional 12-lead ECG waveforms, we employ early fusion by stacking the frequency-time representations of the ECG leads. Additionally, we use recurrent neural networks to exploit the temporal information available in long ECG waveforms. For validation, we have used a large and private collection of ECG waveforms of more than 15,000 MI patients. For comparative purposes, 
we have also validated our proposed model on the publicly available and commonly used PTB Diagnostic ECG database [11].

The remainder of this paper is organised as follows: Section 2 formulates the problem and presents the proposed spectral-longitudinal framework and describes its components in detail. Section 3 describes the experiments, that is the datasets considered and model parameter setup, as well as the results obtained and discussion. Finally, concluding remarks are presented in Section 4.

\section{Our Approach}

In this section, we present our end-to-end machine learning pipeline to model and predict MI diagnosis from 12-lead ECG waveforms, by circumventing the need to manually craft features.

\subsection{Overview}

Figure 2 shows the overview of our proposed approach. Let $\mathcal{D}$ denote a dataset that contains the 12-lead ECG waveforms of $N$ patients who have been tested for MI, i.e., $\mathcal{D}=\left\{R_{i}\right\}_{i=1}^{N}$, we develop an ML binary classification algorithm to identify the subset of patients with MI, from those who do not have MI. The 12 leads are composed of the six limb leads $(I, I I, I I I, a V R, a V L$, and $a V F)$ and the six precordial leads $\left(V_{1}, \cdots, V_{6}\right)$, as illustrated in Fig. 1 (a)). Thus, the ECG data of the $i^{t h}$ patient can be expressed as $R_{i}=\left(\mathbf{r}_{i}^{1}, \cdots, \mathbf{r}_{i}^{12}\right)$. The proposed approach outputs $s_{i}$, which is the prediction probability of a patient being diagnosed with MI. We also employ early fusion of information from multiple leads, by combining or stacking their spectrograms similarly to how a clinician would view a 12 lead ECG trace (as shown in Fig. 1 (b)) which is then followed by either a spectral $\mathcal{S}(\cdot)$, longitudinal $\mathcal{L}(\cdot)$ or joint spectral-longitudinal $\Psi(\cdot)$ model.

The pre-processing step concerns removing noise and movement artefacts from the ECGs, using a band-pass filter. Furthermore, an overlapping window was applied to generate samples from each lead waveform. Given a time series of ECG waveform for a particular lead, $\mathbf{r}_{i}^{l} \in R_{i}$, we extracted multiple samples (windows) from each lead in a patient, i.e., $\mathbf{r}_{i}^{l}=\left(\mathbf{u}_{i 1}^{l}, \mathbf{u}_{i 2}^{l}, \cdots, \mathbf{u}_{i w}^{l}, \cdots, \mathbf{u}_{i W}^{l}\right)$, where $W$ is the number of windows extracted from $\mathbf{r}_{i}^{l}$. Note that for the purposes of readability, we hereafter drop the subscript $i$.

We then employ a fast Fourier transform, $\mathcal{T}(\cdot)$, to map the ECG waveform, $\mathbf{t}_{w}^{l}$, into a frequency-time representation as a spectrogram, i.e. $\Omega_{w}^{l}=\mathcal{T}\left(\mathbf{t}_{w}^{l}\right)$ ) (see Fig. 3). A spectrogram encodes the frequency-time characteristics of the ECG waveforms, and it is hypothesised that a spectrogram of an MI ECG would exhibit different patterns than that from a healthy subject. Another advantage of using a spectral-based data representation is the generalisation it can offer, as it would eliminate variations in ECG device specifications such as sampling rates and mounting positions [17]. Furthermore, the 2-D representation of spectrograms produces a visualisation of the ECG signals, enabling the application 


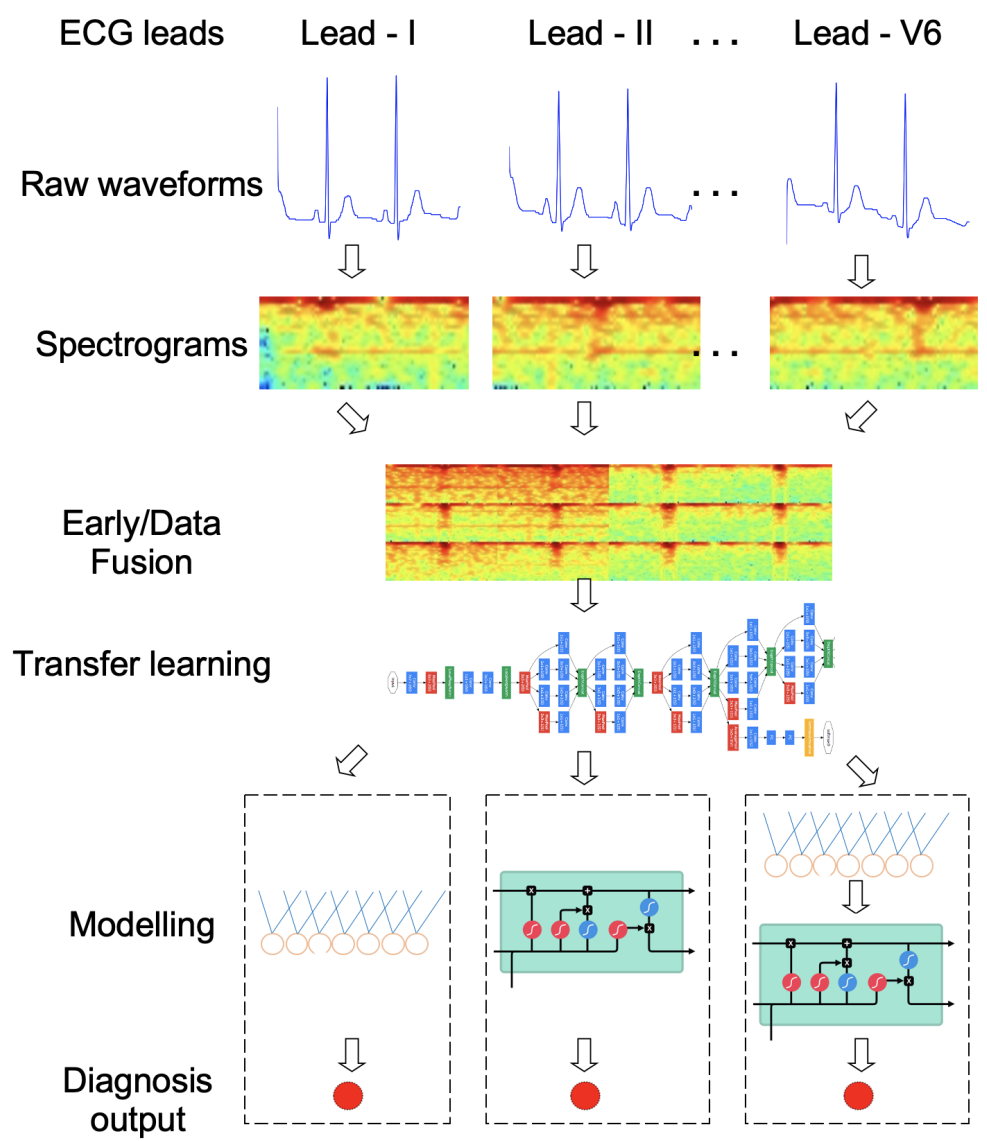

\section{Approach Spectral Longitudinal Spectral-longitudinal}

Fig. 2: Overview of the proposed approach. First, a spectral representation is obtained from each lead followed by early data fusion of these spectrograms via stacking. Next, existing computer vision networks are utilised to extract features from their hidden layers via transfer learning. Finally, modelling is performed by either a spectral, longitudinal or our proposed joint spectral-longitudinal model. 

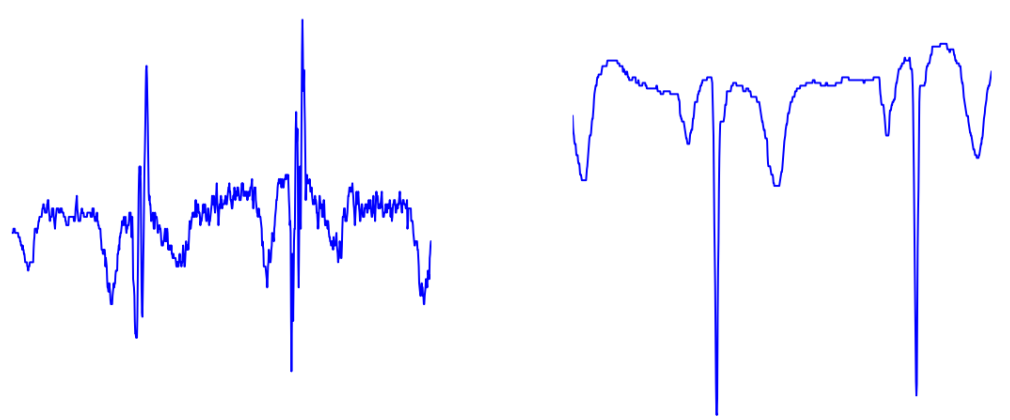

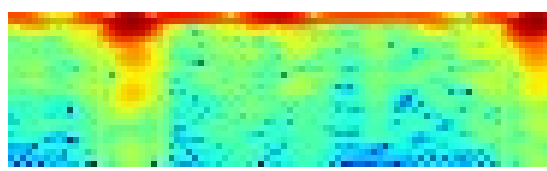

(a) MI

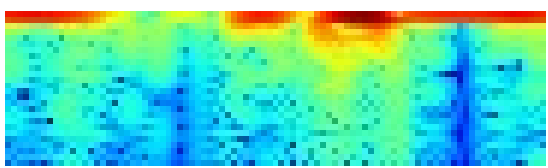

(b) Normal

Fig. 3: Examples of a 2-second aVR lead ECG waveform (top) and its corresponding spectrograms (bottom) for a MI and healthy subject, respectively.

of image-based CNNs architectures. These models are well suited to encode discriminative features from the captured ECGs, and importantly, transfer learning can be employed by taking advantage of powerful pre-trained networks from the computer vision application domain. Thereby reducing the amount of data that would have been required if models were trained from scratch.

Moreover, each ECG lead measures the electrical activity of the heart from different angles or orientations [18]. Consideration of multiple leads therefore offers increased diagnostic information, which could be effectively utilised to better discriminate between MI and non-MI. For this purpose, we employ a datalevel fusion scheme at the early stages of the pipeline as shown in Fig. 2, which avoids the need to model each lead separately. The stacking of spectrograms for the different leads has been motivated by the standard ECG manufacture format as shown in Fig. 1 (a). Moreover, early data fusion enables feature encoding step to learn joint features from multiple leads. The output of the early fusion is represented by $\lambda_{w}$ :

$$
\Lambda_{w}=\left[\begin{array}{cc}
\Omega_{w}^{I}, & \Omega_{w}^{a V R}, \Omega_{w}^{V 1}, \Omega_{w}^{V 4} \\
\Omega_{w}^{I I}, & \Omega_{w}^{a V L}, \Omega_{w}^{V 2}, \Omega_{w}^{V 5} \\
\Omega_{w}^{I I I}, & \Omega_{w}^{a V F}, \Omega_{w}^{V 3}, \Omega_{w}^{V 6}
\end{array}\right],
$$

where $\Lambda_{w}$ is the output of the early fusion.

Finally, after early fusion is performed, spectral modelling $-\mathcal{S}(\cdot)$, is applied through the use of CNNs to encode the frequency-time characteristics of the stacked spectrogram - $\Lambda_{w}$. We propose employing cross-domain transfer learning 
by using existing computer vision networks, e.g., GoogLeNet [19], to encode dense features taken from their hidden layers, i.e. $\mathbf{t}_{w}=\mathcal{S}\left(\Lambda_{w}\right)$. This helps to avoid training a dedicated spectral-based CNN from scratch, which is a process that requires enormous amount of training data, computational resources and time.

\subsection{Modelling spectral and longitudinal characteristics}

A high-dimensional feature vector, $\mathbf{t}_{w} \in \mathbb{R}^{\kappa}$, is obtained for each sample (stacked spectrogram of multiple-leads) using GoogLeNet via transfer learning. This feature vector is then served as input to the classification model to provide prediction of the diagnosis. Three approaches of the classification model will be discussed below: Spectral, Longitudinal and Spectral-longitudinal.

Spectral model $-\mathcal{S}(\cdot)$, takes $\mathbf{t}_{w}$ as input and only involves a dense layer (with $\kappa$ neurons) followed by the softmax layer to provide the diagnosis output. The dense layer projects $\mathbf{t}_{w} \in \mathbb{R}^{\kappa}$ into $\mathbf{l}_{w} \in \mathbb{R}^{\tau}$, where $\tau<\kappa$ as

$$
\mathbf{l}_{w}=\sigma\left(W_{l t} \mathbf{t}_{w}+\mathbf{b}_{l}\right),
$$

where $\sigma(\cdot)$ is an element-wise activation function, $W_{l t} \in \mathbb{R}^{\tau \times \kappa}$ is the weight matrix associated with the dense layer and the deep feature $\mathbf{t}_{w}$, and $\mathbf{b}_{l} \in \mathbb{R}^{\tau}$ is a bias vector. Finally, a sigmoid-based output layer is employed that provides the prediction probability vector $s_{w} \in \mathbb{R}$ as

$$
s_{w}=\frac{e^{W_{s l} \mathbf{l}_{w}}}{e^{W_{s l} \mathbf{l}_{w}}+1},
$$

where $W_{s l} \in \mathbb{R}^{1 \times \tau}$ is the weight matrix associated with the softmax layer and the dense layer output $\mathbf{l}_{w}$.

During training, the binary cross entropy loss, $L_{\beta}$, is applied to iteratively minimise the classification error as

$$
L_{\beta}=-\frac{1}{\beta} \sum_{w=1}^{\beta} y_{w} \times \log \left(s_{w}\right)+\left(1-y_{w}\right) \times \log \left(1-s_{w}\right),
$$

where $\beta$ is the number of samples in a specific batch, and $y_{w}$ is the ground truth label associated with the $w^{t h}$ sample.

Longitudinal model $-\mathcal{L}(\cdot)$, is an alternative approach to the spectral model, providing the prediction probability of MI diagnosis from the deep feature representation of a sample $\mathbf{t}_{w}$. However, different from the spectral model, $\mathcal{S}(\cdot)$; $\mathcal{L}(\cdot)$ utilises the temporal dependency existing among subsequent windows generated from a patient, i.e., $\mathbf{t}_{1}, \mathbf{t}_{2}, \cdots, \mathbf{t}_{w}, \cdots, \mathbf{t}_{W}$. To do so, a recurrent neural network (RNN), particularly a long-short term memory (LSTM), is employed to encode the temporal information. LSTM utilises multiple gates (input, forget, 
and output) to control the information stored in the cell memory to mitigate the vanishing gradient problem embedded in vanilla RNN networks. The deep feature representations of consecutive samples are fed into the LSTM, which can control information flow using the gates it contains. Namely, the cell state gate $\mathbf{c}_{w}$ controls the flow from the current input and the previous and current hidden state (denoted by $\mathbf{t}_{w}, \mathbf{h}_{w-1}$ and $\mathbf{h}_{w}$ respectively). Additionally, the contribution of the previous state information, $\mathbf{c}_{w-1}$ is controlled by the forget gate $\mathbf{f}_{w}$. The input gate, $\mathbf{i}_{w}$, naturally controls the new candidate cell state, $\overline{\mathbf{c}}_{w}$. Finally, the output gate $\mathbf{o}_{w}$, evaluates $\mathbf{c}_{w}$ in order to predict the current hidden state, $\mathbf{h}_{w}$ as

$$
\mathbf{h}_{w}=\mathbf{o}_{w} \odot \phi\left(\mathbf{f}_{w} \odot \mathbf{c}_{w-1}+\mathbf{i}_{w} \odot \overline{\mathbf{c}}_{w}\right),
$$

where $\odot$ is an element-wise multiplication and $\phi$ is a tanh activation function. $\mathbf{h}_{w}, \mathbf{i}_{w}, \mathbf{c}_{w}, \mathbf{c}_{w-1}, \overline{\mathbf{c}}_{w}$ and $\mathbf{o}_{w} \in \mathbb{R}^{\eta}$. To obtain the MI diagnosis prediction, a sigmoid-based wrapper is applied on the hidden layer output of the LSTM, $\mathbf{h}_{n}$, similarly to Equation (2), upon which a cross-entropy loss in Equation (3) is applied to train the model.

Spectral-longitudinal model - $\Psi(\cdot)$, comprises both the spectral and longitudinal models discussed above in cascading fashion. Note that separate use of spectral and longitudinal models each poses distinct challenges for effective modelling, i.e., the former model is unable to account for the obvious temporal dependencies that would be present among consecutive samples; on the other hand, though the longitudinal model is capable of encoding such temporal information, training of the LSTM model is often associated with computational challenges and introduction of performance bias as a result of taking the highdimensional $\mathbf{t}_{w}$ as its input. Hence, to circumvent these individual limitations, we propose the joint spectra-longitudinal model, $\Psi(\cdot)$, which addresses the above challenges by jointly utilising the advantages provided by these two separate model paradigms.

Given a subsequent of deep feature vectors as $\mathbf{t}_{w} \in \mathbb{R}^{\kappa}, \Psi(\cdot)$ first employs a dense layer as in Equation (1) resulting $\mathbf{l}_{w} \in \mathbb{R}^{\tau}$. Then an LSTM network is employed to encode the temporal dependency similarly to Equation (4). Note the input to the LSTM is output of the dense layer, $\mathbf{l}_{w}$, not $\mathbf{t}_{w}$. The hidden layer prediction of the LSTM is then wrapped to provide the MI diagnosis prediction vector $s_{w}$ using the sigmoid-based wrapped in Equation (2). The cross-entropy loss function as in Equation (3) is used to jointly train the spectral and longitudinal components of $\Psi(\cdot)$ model.

\section{Experiments}

The proposed discriminant knowledge extraction for MI detection using deep learning is validated in both private and public datasets. This section presents the details of these datasets followed by the set up of parameters used in the 
spectral and longitudinal models. Finally, the result of the proposed method is presented across the two datasets and modelling techniques.

\subsection{Datasets}

We used two datasets, GCI (proprietary) and PTB (public), to validate the proposed spectral-longitudinal model. The GCI dataset contains 10-second 12-lead ECG waveforms from 11,853 MI and 5,528 Normal (a total of 17,381) records with a sampling rate of $500 \mathrm{~Hz}$, collected from the Provincial Key Laboratory of Coronary Heart Disease, Guangdong Cardiovascular Institute (GCI). Similarly, the PTB Diagnostic ECG database contains collected 12-lead ECGs from patients diagnosed with multiple heart diseases, sampled at $1000 \mathrm{~Hz}[11,7]$. Here we consider cases which relate to MI (148 subjects) and health ( 52 subjects) with a total of 200 subjects. As the duration of ECG data may vary across subjects, we used only the first 10-second segment of each patient.

\subsection{Setup}

In the pre-processing step, a moving window with duration of $1 \mathrm{~s}$ with a $50 \%$ overlap is applied to each ECG lead, resulting a total of 19 samples extracted from a $10 \mathrm{~s}$ ECG lead signal. In the spectrogram generation step, a short time Fourier transform (STFT) is applied with a chunk duration of $0.1 \mathrm{~s}$ with $90 \%$ overlap in order to obtain smooth frequency-time representation. In the transfer learning step of exploiting existing computer vision networks, we use the GoogLeNet [19] to encode features from the stacked spectrograms. Particularly, the penultimate hidden layer of GoogLeNet (Inception - v3) framework is used that provides a feature of dimension $\kappa=2,048$. The dense layer in the spectral model is designed with 16 neurons. Similarly, the longitudinal model is designed to be simple with a single-layered LSTM network of 8 neurons at the input, output, and forget gates. The temporal duration of the LSTM is set to 19 samples equiavalent to the number of windows generated from a $10 \mathrm{~s}$ patient signal. During the training of the joint spectral and longitudinal model, we employ a learning rate of 0.001 with a batch size of 76 , which contains two patients per class in each iteration. A five-fold stratified cross-validation is applied to each dataset to partition the data into train and test sets. Area under the receiver operating curve (AUROC), Sensitivity and Specificity are employed as evaluation metrics to compare the performance of MI detection averaged across the five-folds. Moreover, confusion matrices are also presented, when necessary, to assess the misclassification error of MI cases.

\subsection{Results and Discussion}

The area under receiver operating characteristic curve (AUROC) values obtained for the detection of MI in the two validation datasets (GCI and PTB) are shown in Table 1. The spectral, $\mathcal{S}(\cdot)$, and longitudinal, $\mathcal{L}(\cdot)$, models as baseline methods 

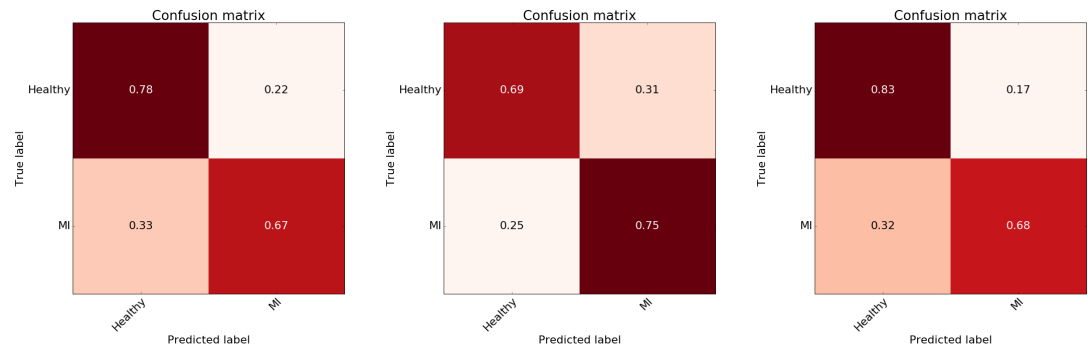

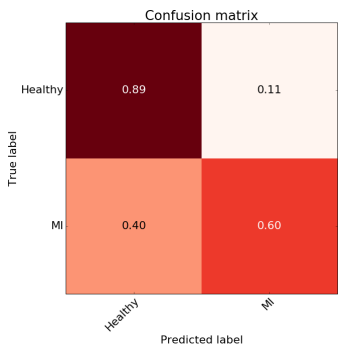

(a) Spectral

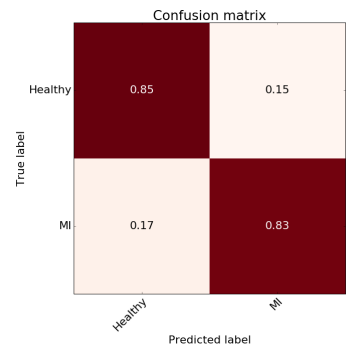

(b) Longitudinal

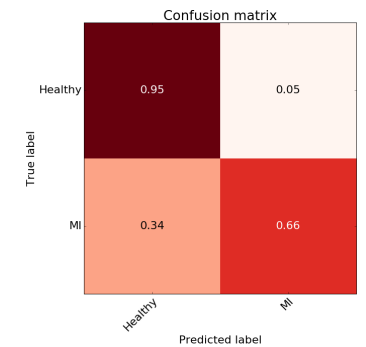

(c) Spectral-longitudinal

Fig. 4: Confusion matrices of spectral, longitudinal and the proposed spectrallongitudinal models. Top row: GCI results; bottom row: PTB results. Higher MI detection performance in the PTB deataset is partially due to the simplicity of the dataset.

are compared with the joint spectral-longitudinal, $\psi(\cdot)$, model. As expected, the baseline methods have inferior performance when compared to the joint spectrallongitudinal model proposed in this work, where the $\psi(\cdot)$ achieves the highest AUROC values of $85 \%$ and $94 \%$ on the GCI and PTB datasets, respectively. The higher classification performance on the PTB dataset can be attributed to the simplicity of MI cases in this dataset, whereas there is a higher degree of patient variation in the GCI dataset. Furthermore, GCI MI cases are different in their onset time, i.e., acute, recent and old, which increases the variation in patient samples even further.

The longitudinal model, $\mathcal{L}(\cdot)$, alone can not achieve significant improvement over the spectral model, i.e., $80 \%$ vs. $81 \%$ in the GCI dataset and $88 \%$ vs. $90 \%$ in the PTB dataset. This can likely be attributed to the longitudinal model overfitting when taking the high dimensional $\left(\mathbf{R}^{2048}\right)$ inception feature vector $\left(\mathbf{t}_{w}\right)$, extracted from GoogleNet, as input. Further evidence of this is provided by the confusion matrices shown in Fig. 4, which can be used to analyse in more detail the misclassification rates of MI cases in the two datasets. The longitudinal models achieve the highest detection sensitivity of the MI cases (75\% in GCI and $83 \%$ in $\mathrm{PTB}$ ). However, this performance comes at the cost of lowest precision (84\% in GCI and $96 \%$ in PTB) and specificity particularly in the GCI dataset 
Table 1: Precision, Sensitivity, Specificity and Area under receiver operating characteristics, AUROC (\%), results of the proposed framework for MI detection validated on the private GCI and public PTB datasets.

GCI Dataset

\begin{tabular}{|c|c|c|c|c|}
\hline Methods & Precision & nsitiv & ecificity & AUROC \\
\hline Spectral & $87 \%$ & $67 \%$ & $78 \%$ & $81 \%$ \\
\hline Longitudinal & $84 \%$ & $\mathbf{7 5} \%$ & $69 \%$ & $80 \%$ \\
\hline Spectral-longitudinal & $\mathbf{9 0} \%$ & $68 \%$ & $83 \%$ & $85 \%$ \\
\hline
\end{tabular}

PTB Dataset

\begin{tabular}{|c|c|c|c|c|}
\hline Methods & Precision & ensitivi & secificity & AUROC \\
\hline Spectral & $\mathbf{9 9} \%$ & $60 \%$ & $89 \%$ & $88 \%$ \\
\hline Longitudinal & $96 \%$ & $83 \%$ & $85 \%$ & $90 \%$ \\
\hline Spectral-longitudinal & $98 \%$ & $66 \%$ & $95 \%$ & $\mathbf{9 4} \%$ \\
\hline
\end{tabular}

(69\% Specificity). By contrast, the joint spectral-longitudinal model achieves the highest AUROC with an effective trade-off between sensitivity and specificity, by using the dense layer outputs of the spectral models to reduce the high feature dimension of the inception features from 2048-D to 8-D. This approach reduces the overfitting issue which hinders the longitudinal models, and consequently the highest specificity values are achieved using spectral-longitudinal models (83\% in GCI and 95\% in PTB). As a result, spectral-longitudinal models are able to achieve the lowest misclassification rate of healthy cases to MI, with only $17 \%$ in GCI - compared to the $22 \%$ and $31 \%$ when using spectral and longitudinal models, respectively. Similarly, only a $5 \%$ misclassification rate of healthy cases to MI occurs in PTB using longitudinal model compared to $11 \%$ using spectral and $15 \%$ using longitudinal models. Similar pattern of reducing the misclassifications of MI cases to Healthy cases occurs, particularly for PTB dataset as the spectral-longitudinal model reduced the MI misclassification by $6 \%$ in PTB compared to the Spectral model. The misclassification rate of MI cases was not reduced significantly in GCI datasets compared to the PTB dataset, due to the higher imbalance and variation of these cases in the former.

\section{Conclusions}

Heart diseases (HDs), and myocardial infarction (MI) in particular, continue to be responsible for millions of deaths worldwide. Clinically, inspection of electrocardiograms (ECGs) by the cardiologists has become acceptable and common practice to provide screening of MI. However, the inter-subject variability associated with ECG interpretation poses a challenge, in addition to the cumbersomeness of the inspection for many patients and longer ECG recordings. These problems are most pronounced in low-resourced environments, in which there are an insufficient number of trained clinical professionals. In this context, machine 
learning algorithms can be employed to assist in the diagnosis process, by providing a data-driven decision support tool to healthcare professionals. While most existing methods that automate HD diagnosis utilise domain specific features, e.g. morphological changes in ECGs, they often do not generalise well across variation in patient characteristics and device specifications. In this paper, we propose an end-to-end deep learning system to detect MI cases from healthy patients using a transfer learning approach and early fusion of multiple leads. The former allows the need for large amounts of training data to be avoided, whilst the latter enables the information across all 12 leads to effectively be exploited through joint-feature encoding. The proposed framework was validated on both private ( $>15,000$ patients) and public datasets and encouraging performance is achieved. Generally, the spectral-longitudinal model that encodes deep spectral representations as well as the temporal dependency in ECG signals, achieved the highest performance over the baseline spectral or longitudinal models on both datasets. Future work includes the exploration of more efficient integration of multiple-leads, as well as identifying the time onset of a heart attack.

\section{Acknowledgements}

This project was supported by the EPSRC "FAST" Healthcare NetworkPlus initiative. TZ was supported by the RAEng Engineering for Development Research Fellowship.

\section{References}

1. Abubakar, S.M., Saadeh, W., Altaf, M.A.B.: A wearable long-term single-lead ecg processor for early detection of cardiac arrhythmia. In: 2018 Design, Automation \& Test in Europe Conference \& Exhibition (DATE). pp. 961-966. IEEE (2018)

2. Acharya, U.R., Fujita, H., Oh, S.L., Hagiwara, Y., Tan, J.H., Adam, M.: Application of deep convolutional neural network for automated detection of myocardial infarction using ECG signals. Information Sciences 415, 190-198 (2017)

3. Al Rahhal, M.M., Bazi, Y., AlHichri, H., Alajlan, N., Melgani, F., Yager, R.R.: Deep learning approach for active classification of electrocardiogram signals. Information Sciences 345, 340-354 (2016)

4. Ansari, S., Farzaneh, N., Duda, M., Horan, K., Andersson, H.B., Goldberger, Z.D., Nallamothu, B.K., Najarian, K.: A review of automated methods for detection of myocardial ischemia and infarction using electrocardiogram and electronic health records. IEEE Reviews in Biomedical Engineering 10, 264-298 (2017)

5. Baloglu, U.B., Talo, M., Yildirim, O., San Tan, R., Acharya, U.R.: Classification of myocardial infarction with multi-lead ecg signals and deep cnn. Pattern Recognition Letters 122, 23-30 (2019)

6. Bax, J.J., Baumgartner, H., Ceconi, C., Dean, V., Fagard, R., Funck-Brentano, C., Hasdai, D., Hoes, A., Kirchhof, P., Knuuti, J., et al.: Third universal definition of myocardial infarction. Journal of the American College of Cardiology 60(16), 1581-1598 (2012) 
7. Bousseljot, R., Kreiseler, D., Schnabel, A.: Nutzung der ekg-signaldatenbank cardiodat der ptb über das internet. Biomedizinische Technik/Biomedical Engineering 40(s1), 317-318 (1995)

8. Darmawahyuni, A., Nurmaini, S., Caesarendra, W., Bhayyu, V., Rachmatullah, M.N., et al.: Deep learning with a recurrent network structure in the sequence modeling of imbalanced data for ecg-rhythm classifier. Algorithms 12(6), 118 (2019)

9. Dash, S., Chon, K., Lu, S., Raeder, E.: Automatic real time detection of atrial fibrillation. Annals of Biomedical Engineering 37(9), 1701-1709 (2009)

10. Duong, H.T.H., Tadesse, G.A., Nhat, P.T.H., Van Hao, N., Prince, J., Duong, T.D., Kien, T.T., Pugh, C., Loan, H.T., Chau, N.V.V., et al.: Heart rate variability as an indicator of autonomic nervous system disturbance in tetanus. The American journal of tropical medicine and hygiene 102(2), 403-407 (2020)

11. Goldberger, A.L., Amaral, L.A., Glass, L., Hausdorff, J.M., Ivanov, P.C., Mark, R.G., Mietus, J.E., Moody, G.B., Peng, C.K., Stanley, H.E.: PhysioBank, PhysioToolkit, and PhysioNet: components of a new research resource for complex physiologic signals. Circulation 101(23), e215-e220 (2000)

12. Goldberger, A.L., Gold-berger, E.: Clinical electrocardiography, a simplified approach. Critical Care Medicine 9(12), 891-892 (1981)

13. Han, C., Shi, L.: Ml-resnet: A novel network to detect and locate myocardial infarction using 12 leads ecg. Computer methods and programs in biomedicine 185, 105138 (2020)

14. Kumar, M., Pachori, R., Acharya, U.: Automated diagnosis of myocardial infarction ecg signals using sample entropy in flexible analytic wavelet transform framework. Entropy 19(9), 488 (2017)

15. Litjens, G., Kooi, T., Bejnordi, B.E., Setio, A.A.A., Ciompi, F., Ghafoorian, M., Van Der Laak, J.A., Van Ginneken, B., Sánchez, C.I.: A survey on deep learning in medical image analysis. Medical Image Analysis 42, 60-88 (2017)

16. Mehta, S., Lingayat, N., Sanghvi, S.: Detection and delineation of P and T waves in 12-lead electrocardiograms. Expert Systems 26(1), 125-143 (2009)

17. Ravi, D., Wong, C., Lo, B., Yang, G.Z.: A deep learning approach to on-node sensor data analytics for mobile or wearable devices. IEEE Journal of Biomedical and Health Informatics 21(1), 56-64 (2017)

18. Strodthoff, N., Strodthoff, C.: Detecting and interpreting myocardial infarction using fully convolutional neural networks. Physiological Measurement (2018)

19. Szegedy, C., Liu, W., Jia, Y., Sermanet, P., Reed, S., Anguelov, D., Erhan, D., Vanhoucke, V., Rabinovich, A.: Going deeper with convolutions. In: Proceedings of the IEEE Conference on Computer Vision and Pattern Recognition. pp. 1-9 (2015)

20. Tadesse, G.A., Javed, H., Thanh, N.L.N., Thai, H.D.H., Le Van, T., Thwaites, L., Clifton, D., Zhu, T.: Multi-modal diagnosis of infectious diseases in the developing world. IEEE Journal of Biomedical and Health Informatics (2020)

21. Tadesse, G.A., Javed, H., Weldemariam, K., Zhu, T.: A spectral-longitudinal model for detection of heart attack from12-lead electrocardiogram waveforms. In: Proc. of Annual International Conference of the IEEE Engineering in Medicine and Biology Society (EMBC). p. to appear (2020)

22. Tadesse, G.A., Zhu, T., Liu, Y., Zhou, Y., Chen, J., Tian, M., Clifton, D.: Cardiovascular disease diagnosis using cross-domain transfer learning. In: Proc. of Annual International Conference of the IEEE Engineering in Medicine and Biology Society (EMBC). pp. 4262-4265 (2019) 
23. Tadesse, G.A., Zhu, T., Thanh, N.L.N., Hung, N.T., Duong, H.T.H., Khanh, T.H., Quang, P.V., Tran, D.D., Yen, L.M., Doorn, H.R.V., andJohn Prince, N.V.H., Javed, H., Kiyasseh, D., Tan, L.V., Thwaites, L., Clifton, D.A.: Severity detection tool for patients with infectious disease. arXiv preprint arXiv:1912.05345 (2019)

24. (WHO), W.H.O.: Cardiovascular diseases (CVDs). URL link: www.who.int/newsroom/fact-sheets/detail/cardiovascular-diseases-(cvds), Last accessed on 13 August 2020 